\title{
Synthesis, Characterization and Biological activity of new derivatives of Chromen-2-one
}

\author{
Aziz Behrami \\ Public University of Mitrovica, Faculty of Food Technology, Kosovo, PIM Trepca-40000, Mitrovice, Kosovo
}

\begin{abstract}
We report the organic syntheses of new derivatives from Chromen-2-one and their antibacterial activity. Compounds 4-[Acetyl-(2-oxo-2H-chromen-4-yl)-amino]-benzenesulfonyl chloride 1a, 4-[Acetyl-(2oxo-3-phenylsulfamoyl-2H-chromen-4-yl)-amino]-benzenesulfonyl chloride 2a ,2-\{4-[Acetyl-(4-chlorosulfonylphenyl)-amino]-2-oxo-3-phenylsulfamoyl-2H-chromen-7-ylamino\}-benzoic acid 3a. All Structures have been synthesized and characterized using melting points, IR spectra, ${ }^{1} \mathrm{H}-\mathrm{NMR},{ }^{13} \mathrm{C}$-NMR spectra, and elemental analyses. The purified synthesized compounds $(\mathbf{1 a}, 2 \boldsymbol{2 a}, \mathbf{3 a})$, at contcentrations $2,3,5 \mathrm{mg} / \mathrm{ml}$ was tested for their antibacterial activity against three bacterial cultures ;Staphylococcus aureus, Escherchia coli and Bacillus cereus. The antibacterial activity of synthesized compounds are compared with antibacterial activity of standard antibiotics cephalexine and streptomycine.
\end{abstract}

The compounds (1a, 2a, 3a) shows different bacteriostatic and bacteriocidal activity.

Keywords: Chromen-2-one derivatives, streptomycine

\section{Introduction}

Starting from Chromen-2-one (a); are synthesized some new derivatives 1a, 2a, 3a (Schemes 1, 2, 3). Coumarine derivatives are large group of heterocyclic with oxygen as heteroatom.Coumarine is a chemical compound (specifically, a benzo- $\alpha$-pyrone) found in many plants notably in high concentration in the tonka bean (Dipteryx odorata), vanilla grass (Anthoxanthum odoratum), woodruff (Galium odoratum), mullein (Verbascum spp), and sweet grass (Hierochloe odorata).Coumarine and their derivatives have shown various biological activities. Other several coumarin derivatives have antimicrobial properties (Sanghyun; et al 1996; Mohareb et al 2007; Nofal et al 2000), with reflux and condensation we have synthesize some new coumarine derivatives and to investigate their antibacterial activity against Staphylococcus aureus, E.coli and Bacillus cereus.The antibacterial activity of synthesized compounds is compared with antibacterial activity of Cefalexine and Streptomycine.

\section{Experimental Chemistry}

\section{Material And Methods}

Compounds 4-[Acetyl-(2-oxo-2H-chromen-4-yl)-amino]-benzenesulfonyl chloride 1a,

4-[Acetyl-(2-oxo-3-phenylsulfamoyl-2H-chromen-4-yl)-amino]-benzenesulfonyl chloride 2a, 2-\{4-[Acetyl-(4chlorosulfonyl-phenyl)-amino]-2-oxo-3-phenylsulfamoyl-2H-chromen-7-ylamino\}-benzoic acid 3a. Are synthesized.

\section{Measurement}

The identification of Chromen-2-one derivatives (1a,2a,3a,) , is made by using melting point, IR , ${ }^{1} \mathrm{H}$ NMR , ${ }^{13} \mathrm{C}$-NMR spectra and elemental analysis.

Melting point was determinated on an electrothermal apparatus (Fisher Scientific 2555) in a open capillary tube and wa not corrected. Infrared spectra were recorded in $\mathrm{cm}-1$ for $\mathrm{KBr}$ pellts on a FT-IR Shimadzu 8400 S spectrophotometer with resolution $4 \mathrm{~cm}-1,{ }^{1} \mathrm{H}$ - NMR spectra were recorded on a Bruker UNITY plus-500 'NMR 1' spectrometer using DMSO-d6 as the solvent and TMS as the internal references standard ( $\sigma=0,00$ ppm). Chemical shifts are expressed in $\delta$ ppm. Mass spectra were taken on a LKB 9000 mass spectrometer. Element analysze was performed on a Perikin-Elmer 240 BCHN analyzer. The purity of the compounds (synthesized) was routinely checked by TLC using Merck Kieselgel-60 (F-254) and benzene,toluene,glacial acetic acid (80:10:10)as mobile phase. The spots were exposed in iodine vapour for visualization.

Preparation of 4-[Acetyl-(2-oxo-2H-chromen-4-yl)-amino]-benzenesulfonyl chloride (1a)

For this synthesis is used $5 \mathrm{~g}$ Chromen-2-one as substrate, $5 \mathrm{~g}$ N-Phenyl-acetamide, and $0.5 \mathrm{ml} \mathrm{HCl}, 5 \mathrm{~mL}$ $\mathrm{HSO}_{3} \mathrm{CL}, 10 \mathrm{~mL} \mathrm{CH}_{3} \mathrm{CN}, 1 \mathrm{~mL} \mathrm{Et}_{3} \mathrm{~N}$ as catalyzer.

The mixture was refluxed at $65^{\circ} \mathrm{C}$ for ca. $1 \mathrm{~h}$.

The obtained crystals are filtred and rinsed with $\mathrm{CH}_{3} \mathrm{CN}$ and dried at room temperature. Recrystallization form absolute $\mathrm{CH}_{3} \mathrm{CN}$ gave a yellow product of $\left(80 \%\right.$ yield, melting point $298{ }^{\circ} \mathrm{C}$. 


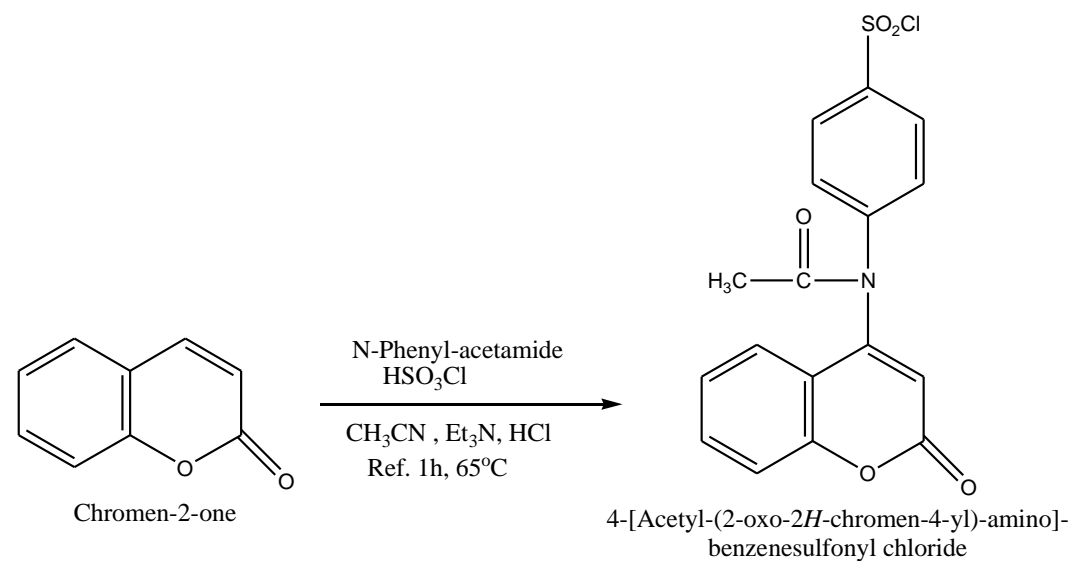

Figure 1. 4-[Acetyl-(2-oxo-2H-chromen-4-yl)-amino]-benzenesulfonyl chloride (1a)

Preparation of 4-[Acetyl-(2-oxo-3-phenylsulfamoyl-2H-chromen-4-yl)-amino]- benzenesulfonyl chloride (2a) In a $100 \mathrm{ml}$ flask were mixed 3g 4-[Acetyl-(2-oxo-2H-chromen-4-yl)-amino]-benzenesulfonyl chloride with 6 $\mathrm{ml}$ phenylamine, $3 \mathrm{~mL} \mathrm{H}_{2} \mathrm{SO} 4,2 \mathrm{~mL} \mathrm{H}_{2} \mathrm{~S}_{2} \mathrm{O}_{7}, 10 \mathrm{~mL} \mathrm{CH}_{3} \mathrm{CN}$ and $1 \mathrm{~mL} \mathrm{Et}_{3} \mathrm{~N}$, the mixture was refluxed at 160 ${ }^{\circ} \mathrm{C}$ for ca. $1 \mathrm{~h}$

The obtained crystals are filtred and dried at room temperature. Recrystallization form $\mathrm{CH}_{3} \mathrm{CN}$ gave yellow crystals product of $75 \%$ yield, meltingpoint, and $315^{\circ} \mathrm{C}$.

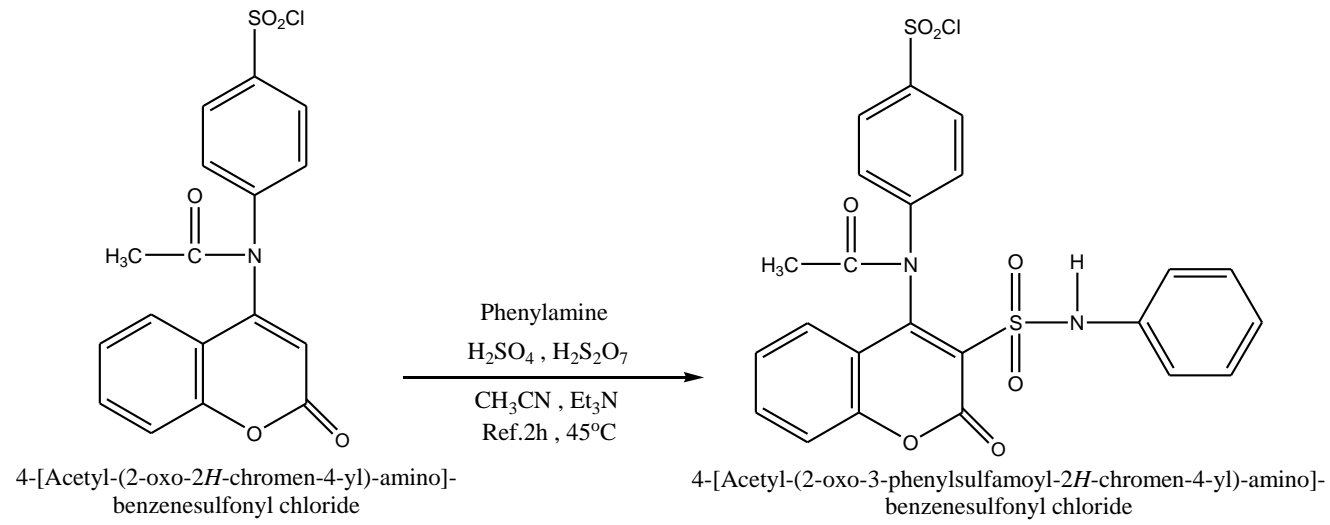

Figure 2: Preparation of 4-[Acetyl-(2-oxo-3-phenylsulfamoyl-2H-chromen-4-yl)-amino]- benzenesulfonyl chloride (2a)

Preparation of 2-\{4-[Acetyl-(4-chlorosulfonyl-phenyl)-amino]-2-oxo-3-phenylsulfamoyl-2H-chromen-7ylamino $\}$-benzoic acid (3a)

In a $100 \mathrm{~mL}$ flask were mixed $2.5 \mathrm{~g}$ of 4-[Acetyl-(2-oxo-3-phenylsulfamoyl-2H-chromen-4-yl)-amino]benzenesulfonyl chloride, with $10 \mathrm{~mL}$ Acetonitrile, $3 \mathrm{~g} 2$-amino benzoic acid, $0.5 \mathrm{~mL} \mathrm{Et}_{3} \mathrm{~N}$ as katalyzer.The mixture was refkuxed at $45{ }^{\circ} \mathrm{C}$ in water bath for ca.2h. After filtration the product was recrystallized from $\mathrm{C}_{2} \mathrm{H}_{5} \mathrm{OH}$. The recrystallizacion gave a red product at $70 \%$ yield, meltingpoint; $322{ }^{\circ} \mathrm{C}$.
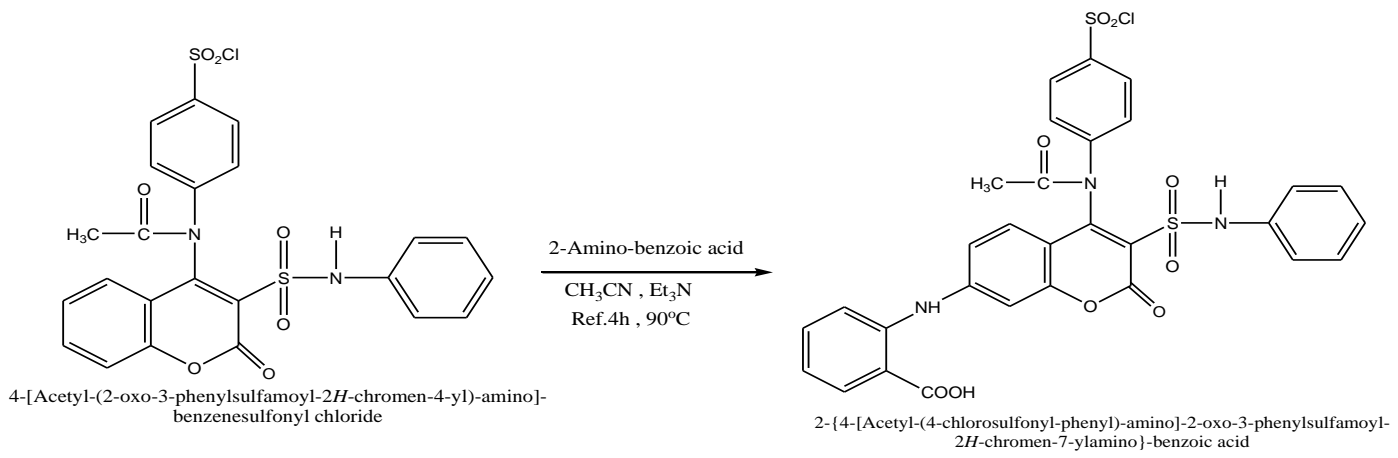

Figure 3: 2-\{4-[Acetyl-(4-chlorosulfonyl-phenyl)-amino]-2-oxo-3-phenylsulfamoyl-2H-chromen-7-ylamino $\}$ benzoic acid (3a) 
Table-1: characteristics and analytical data of the complexes

\begin{tabular}{|l|l|l|l|l|l|l|l|l|l|}
\hline Comp & $\begin{array}{c}\text { Yeld } \\
\end{array}$ & m.p & M.F & & & & & & \\
\cline { 5 - 10 } & & & & C & S & Cl & H & N & O \\
\hline $1 \mathrm{a}$ & 80 & $298^{\circ} \mathrm{C}$ & $\mathrm{C}_{17} \mathrm{H}_{12} \mathrm{ClNO}_{5} \mathrm{~S}$ & 54.05 & 8.49 & 9.38 & 3.20 & 3.71 & 21.17 \\
& & & & 53.92 & 8.45 & 9.30 & 3.10 & 3.68 & 21.14 \\
\hline $2 \mathrm{a}$ & 75 & $315^{\circ} \mathrm{C}$ & $\mathrm{C}_{23} \mathrm{H}_{17} \mathrm{ClN}_{2} \mathrm{O}_{7} \mathrm{~S}$ & 51.82 & 12.01 & 6.65 & 3.21 & 5.26 & 21.01 \\
& & & 51 & 11.94 & 6.60 & 3.18 & 5.21 & 20.90 \\
\hline $3 \mathrm{a}$ & 70 & $322{ }^{\circ} \mathrm{C}$ & $\mathrm{C}_{28} \mathrm{H}_{20} \mathrm{ClN}_{3} \mathrm{O}_{9} \mathrm{~S}_{2}$ & 52.38 & 9.99 & 5.52 & 3.14 & 6.54 & 22.43 \\
& & & 52.0 & 9.94 & 5.49 & 3.12 & 6.52 & 22.39 \\
\hline
\end{tabular}

\section{Antibacterial activity}

The purified synthesized compounds $(1 \mathrm{a}, 2 \mathrm{a}, 3 \mathrm{a}$, ) was subjected to test in vitro its antibacterial activity against three bacterial cultures ; Staphylococcus aureus,E.Coli and B.cereus. Antibacterial activity of compounds was investigated applying the Kirby-Bayer method or disc method (d=5.5 mm max. capacity $10 \mu \mathrm{g})$

Table 2 Antibacterial activity- Staphylococcus aureus Inhibition zone (mm)

\begin{tabular}{|l|l|l|ll|}
\hline Compound & $2 \mathrm{mg} / \mathrm{mL}$ & $3 \mathrm{mg} / \mathrm{mL}$ & $5 \mathrm{mg} / \mathrm{mL}$ \\
\hline $1 \mathrm{a}$ & 11 & 16 & 19 & \\
\hline 2a & 11 & 17 & 18 \\
\hline 3a & 10 & 17 & 17 & $10 \mu \mathrm{g}$ \\
\hline Cefalexine & 9 & 9 & 9 & $10 \mu \mathrm{g}$ \\
\hline Streptomycine & 20 & 20 & 20 & \\
\hline
\end{tabular}

Table 3 Antibacterial activity - E.Coli

Inhibition zone $(\mathrm{mm})$

\begin{tabular}{|c|c|c|c|}
\hline Compound & $2 \mathrm{mg} / \mathrm{mL}$ & $3 \mathrm{mg} / \mathrm{mL}$ & $5 \mathrm{mg} / \mathrm{mL}$ \\
\hline $1 \mathrm{a}$ & 10 & 11 & 17 \\
\hline $2 \mathrm{a}$ & 11 & 12 & 18 \\
\hline $3 a$ & 11 & 13 & 19 \\
\hline Cephalexine & 9 & 9 & $10 \mu \mathrm{g}$ \\
\hline Streptomycine & 23 & 23 & $10 \mu \mathrm{g}$ \\
\hline
\end{tabular}

Table 4 Antibacterial activity - Bacillus cereus Inhibition zone (mm)

\begin{tabular}{|l|l|l|ll|}
\hline Compound & $2 \mathrm{mg} / \mathrm{mL}$ & $3 \mathrm{mg} / \mathrm{mL}$ & $5 \mathrm{mg} / \mathrm{mL}$ & \\
\hline $1 \mathrm{a}$ & 11 & 15 & 21 & \\
\hline 2a & 10 & 15 & 22 & \\
\hline 3a & 12 & 18 & 24 & $10 \mu \mathrm{g}$ \\
\hline Cephalexine & 9 & 9 & 9 & $10 \mu \mathrm{g}$ \\
\hline Streptomycine & 23 & 23 & 23 & \\
\hline
\end{tabular}

\section{Results And Discussion}

By reacting equimolar amounts of Chromen-2-one and correspomding reagents (according scheme 1) under reflux reaction condictions product 1a is synthesized in $80 \%$ yield.

By reacting equimolar amounts of 4-[Acetyl-(2-oxo-2H-chromen-4-yl)-amino]-

Benzenesulfonyl chloride 1a and corresponding reagents (according scheme 2) under reflux reaction condictions product 2a is synthesized in $75 \%$ yield.

By reacting equimolar amounts of 4-[Acetyl-(2-oxo-3-phenylsulfamoyl-2H-chromen-4-yl)-amino]benzenesulfonyl chloride 2a , and corresponding reagents (according scheme 3) under reflux reaction condictions product2-\{4-[Acetyl-(4-chlorosulfonyl-phenyl)-amino]-2-oxo-3-phenylsulfamoyl-2H-chromen-7ylamino $\}$-benzoic acid $\mathbf{3 a}$ is synthesized in $70 \%$ yield.

The structure of Chromen-2-one derivative (1a,2a,3a)were determined fromtheir IR, ${ }^{1} \mathrm{H}$ NMR,${ }^{13} \mathrm{C}$ NMR spectar and their melting points as follows.

For (1a); IR bands $\left(\mathbf{K B r}, \mathbf{c m}^{-1}\right) 3146 \mathrm{~cm}^{-1}$ (C-H stretch. aromatic) ; $3070 \mathrm{~cm}^{-1}$ (C-H stretch. aromatic) ; $2938 \mathrm{~cm}^{-}$ ${ }^{1}$ (C-H stretch. alifatic) ;1680 cm $\mathrm{cm}^{-1}(\mathrm{C}=\mathrm{O}) ; 1600 \mathrm{~cm}^{-1}$ (C=O $\alpha$-pyron) ; $1315 \mathrm{~cm}^{-1}$ (C-O stretch.) ; $1240 \mathrm{~cm}^{-1}$ $\left(\mathrm{SO}_{2} \mathrm{Cl}\right) ; 758 \mathrm{~cm}^{-1}(\mathrm{C}-\mathrm{C}$ aromatic $)$

${ }^{1}{ }^{1}$ NMR (DMSO-d $\mathbf{d}_{6}$ ) $\mathbf{6 p p m}, 7.92 \mathrm{ppm} ; 7.22 \mathrm{ppm} ; 7.45 \mathrm{ppm} ; 7.87 \mathrm{ppm} ; 7.63 \mathrm{ppm} ; 5.88 \mathrm{ppm} ; \mathrm{m}$ (9H.aromatic) ; 2.02ppm s $\left(\mathrm{H}, \mathrm{CH}_{3}\right)$

${ }^{13}$ C NMR (DMSO) $\delta$ ppm , 162.0ppm (C=O, $\alpha$ pyron) ; 162.8ppm (C-N);

$150.8 \mathrm{ppm} ;(\mathrm{C}-\mathrm{O})$; 147.6ppm (C-N); 139.7ppm (C-S) ; 128.1ppm ; 127ppm ; 125.2ppm 121.6ppm(10C aromatic) ; $15.8 \mathrm{ppm}\left(\mathrm{C}, \mathrm{CH}_{3}\right)$ 
For (2a) IR bands $\left(\mathbf{K B r}, \mathbf{c m}^{-1}\right) 3140 \mathrm{~cm}^{-1}\left(\mathrm{C}-\mathrm{H}\right.$ aromatic); $2936 \mathrm{~cm}^{-1}$ (C-H alifatic) ;1680 $\mathrm{cm}^{-1}$ (C=O stretch.) ; $1600 \mathrm{~cm}^{-1}(\mathrm{C}=\mathrm{O} \alpha$ pyron $) ; 1380 \mathrm{~cm}^{-1}(\mathrm{CONH}) ; 1230 \mathrm{~cm}^{-1}\left(\mathrm{SO}_{2} \mathrm{Cl}\right) ; 1210 \mathrm{~cm}^{-1}\left(\mathrm{SO}_{2}-\mathrm{NH}\right) ; 1150 \mathrm{~cm}^{-1}(\mathrm{CONH}) ;$ $750 \mathrm{~cm}^{-1}$ (C-C aromatic)

${ }^{1}$ H NMR (DMSO-d ( $_{\mathbf{6}}$ ) $\mathbf{8 p p m} 7.82 \mathrm{ppm} ; 7.63 \mathrm{ppm} ; 7.45 \mathrm{ppm} ; 7,27 \mathrm{ppm} ; 7.20 \mathrm{ppm} ; 7.01 \mathrm{ppm} ; 6.62 \mathrm{ppm}$; $6.46 \mathrm{ppm} ; \mathrm{m}(13 . \mathrm{H}$ aromatic $) ; 4.0 \mathrm{ppm} \mathrm{s}(\mathrm{H}, \mathrm{NH}) ; 2.09 \mathrm{ppm} \mathrm{s}\left(\mathrm{H}, \mathrm{CH}_{3}\right)$.

${ }^{13}$ C NMR (DMSO) $\delta p p m \quad 162.42 \mathrm{ppm}(\mathrm{C}-\mathrm{N}) ; 160.0(\mathrm{C}, \mathrm{C}=\mathrm{O}) ; 150.8 \mathrm{ppm}(\mathrm{C}-\mathrm{O}) ; 147.7 \mathrm{ppm}(\mathrm{C}-\mathrm{N}) ; 139.7 \mathrm{ppm}$ (C-S);128.1ppm; $127.0 \mathrm{ppm} ; 125.2 \mathrm{ppm} ; 129.3 \mathrm{ppm} ; 118.5 \mathrm{ppm}\left(14 \mathrm{C}\right.$. aromatic ) ; 15.8ppm $\left(\mathrm{C}_{1} \mathrm{CH}_{3}\right)$

For (3a) IR bands $\left(\mathrm{KBr}, \mathrm{cm}^{-1}\right) 3300 \mathrm{~cm}^{-1}\left(\mathrm{O}-\mathrm{H}\right.$, stretch.) ; $3065 \mathrm{~cm}^{-1}\left(\mathrm{C}-\mathrm{H}\right.$,stretch. aromatic); $1680 \mathrm{~cm}^{-1}(\mathrm{C}=\mathrm{O})$; $1720 \mathrm{~cm}^{-1}(\mathrm{C}=\mathrm{O}$,stretch. $) ; 1680 \mathrm{~cm}^{-1}(\mathrm{C}=\mathrm{O}) ; 1320 \mathrm{~cm}^{-1}\left(\mathrm{SO}_{2} \mathrm{Cl}\right) ; 1280 \mathrm{~cm}^{-1}(\mathrm{NH}) ; 740 \mathrm{~cm}^{-1}(\mathrm{C}-\mathrm{C}$ aromatic $)$.

${ }^{1} \mathbf{H}$ NMR (DMSO-d $\mathbf{d}_{\mathbf{6}}$ ) $\mathbf{8 p p m} \quad 11.2 \mathrm{ppm} \mathrm{s}(\mathrm{H}, \mathrm{COOH}) ; 7.92 \mathrm{ppm} ; 7.88 \mathrm{ppm} ; 7.87 \mathrm{ppm} ; 7.35 \mathrm{ppm} ; 7.01 \mathrm{ppm}$; $6.83 \mathrm{ppm} ; 6.46 \mathrm{ppm} ; 6.42 \mathrm{ppm} ; 6.40 \mathrm{ppm} ;(16 . \mathrm{H}$ aromatic $) 4.0 \mathrm{ppm} ; \mathrm{s}(\mathrm{H}, \mathrm{NH}) ; 2.02 \mathrm{ppm} \mathrm{s}\left(\mathrm{H}_{1} \mathrm{CH}_{3}\right)$.

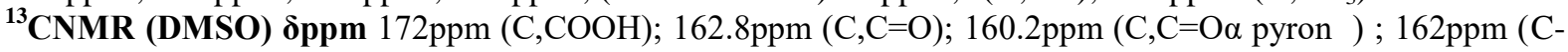
N) ;151.7ppm (C-O) ; 142.8ppm (C-N);139.7ppm (C-S); 134.6ppm ; 129.3ppm ; 127ppm ; $118.5 \mathrm{ppm}$; $117.8 \mathrm{ppm} ; 115.1 \mathrm{ppm} ; 114.6 \mathrm{ppm}$ (17C.aromatic);15.8ppm $\left(\mathrm{C} \mathrm{CH}_{3}\right)$.

From the results the following conclusion where drawn.

\section{Conclusion}

The study provides the first evidence that compounds (1a, 2a, 3a, ) obviously inhibit the growth of S.auerus, E.coli and B.cereus. The compounds (1a, 2a, 3a) compared with the antibacterial activity of Streptomycine in S.aureus, E.coli and B.cereus. This study provided the first evidence that these compounds 1a, 2a, 3a, showed a significant antibacterial effect against S.aureus, E.coli and B.Cereus. The chemical structures of synthesizen compounds were determined according to extensive NMR experiments and published data.

\section{Acknowledgements}

The authors thank Prof.Branko Stanovnik, University of Ljubljana and its laboratory staff for ${ }^{1} \mathrm{H}$ NMR spectrum and elemental analyses.

\section{References}

[1]. $\quad$ S. Govori. , V. Kalaj., V. Rapic. ; L.Kalaj. ; And S.Dakovic. Heterocycel.Commun. 2002; 8,129-134.

[2]. B.Stanovnik.;H. Susachitzky .;E.F Scriven , Progress in Heterocyclic Chemistry, Pergamon Press ,Oxford , 1993 ; 5,75-146.

[3]. S.H.Lee.; D.S.Shin,; J.S. Kim.; K.-B. Oh and S.S .Kan. Arch. Pharm. Res, 2003; 26-32.

[4]. K.B Vyas. .; K.S Nimavat.;G.R. Jani ; and M.V.Hathi..; Orbital, 2009; 1, 183-189.

[5]. A.V.Abyshev; V.A Gimdein.; E.V Semenov.; E.M Agev.; A.A. Abdulla.; Zade and Gueseinov, Pharm.Chem.J., 2006; 40.607-612.

[6]. M.D.Aytemir; R.C. Hider. .; D.D.Erol.; M.Ozalp ; and M.Ekizoglu ; Turk.J.chem., 2003 ; 27 : $445-452$.

[7]. M.M .El.Saghie; M.B. Naili; M.Kh. Rammash..; N.H. Saleh.; and K.M.Kreddan.; Arkivoc, 2007; 83-92.

[8]. Z.M .Nofa; M. El-Zaha and M.Abd El Karim., Molecules, 2000; 5: 99-105.

[9]. K.C. Chaluvaraju; and K.Ishwarbha...Asian. J Chem 2008; 20, 4335-4340.

[10]. R.Ra Kali; S, Grworamma; and B. Suresh.; Asian J Chem 2008: 20: 5289-5293.

[11]. Mohammed Ashraf.A and Mohammed.SH. Boorg Med Chem. Lett 2009; 17: 3314-3320.

[12]. ZM. Nofal; M.El-Zahar; S. Abd El-Karim .Novel, coumarin derivatives with expected biological activity, Molecules. 2000; 5: 99113 